Pacific

Journal of

Mathematics

HOW LIKELY IS BUFFON'S NEEDLE TO FALL NEAR A PLANAR CANTOR SET?

Yuval Peres and Boris Solomyak

Volume $204 \quad$ No. 2

June 2002 


\title{
HOW LIKELY IS BUFFON'S NEEDLE TO FALL NEAR A PLANAR CANTOR SET?
}

\author{
Yuval Peres and Boris Solomyak \\ Dedicated to the memory of Thomas H. Wolff
}

\begin{abstract}
Let $\Lambda$ be a compact planar set of positive finite one-dimensional Hausdorff measure. Suppose that the intersection of $\Lambda$ with any rectifiable curve has zero length. Then a theorem of Besicovitch (1939) states that the orthogonal projection of $\Lambda$ on almost all lines has zero length. Consequently, the probability $p(\Lambda, \epsilon)$ that a needle dropped at random will fall within distance $\epsilon$ from $\Lambda$, tends to zero with $\epsilon$. However, existing proofs do not yield any explicit upper bound tending to zero for $p(\Lambda, \epsilon)$, even in the simplest cases, e.g., when $\Lambda=$ $K^{2}$ is the Cartesian square of the middle-half Cantor set $K$. In this paper we establish such a bound for a class of selfsimilar sets $\Lambda$ that includes $K^{2}$. We also determine the order of magnitude of $p(\Lambda, \epsilon)$ for certain stochastically self-similar sets $\Lambda$. Determining the order of magnitude of $p\left(K^{2}, \epsilon\right)$ is an unsolved problem.
\end{abstract}

\section{Introduction.}

Consider $K=\left\{\sum_{n=1}^{\infty} a_{n} 4^{-n}: a_{n} \in\{0,3\}\right\}$, the middle-half Cantor set, and the direct product $K^{2}=K \times K \subset \mathbb{R}^{2}$. It is well-known that the one-dimensional Hausdorff measure of $K^{2}$ satisfies $0<\mathcal{H}^{1}\left(K^{2}\right)<\infty$ and that $K^{2}$ is totally unrectifiable. Therefore, by Besicovitch's theorem (see [4, Theorem 6.13]), the projection of $K^{2}$ on almost every line through the origin, has zero length. This can be expressed by saying that the Favard length of $K^{2}$ equals zero. Recall (see [2, p. 357]) that the Favard length of a planar set $E$ is defined by

$$
\operatorname{Fav}(E)=\int_{0}^{\pi}\left|\operatorname{proj}_{\theta} E\right| d \theta
$$

where $\operatorname{proj}_{\theta}$ denotes the orthogonal projection from $\mathbb{R}^{2}$ onto the line through the origin making angle $\theta$ with the horizontal axis, and $|A|$ denotes the Lebesgue measure of a measurable set $A \subset \mathbb{R}$. The Favard length of a set $E$ in the unit square has a probabilistic interpretation: Up to a constant factor, it is the probability that "Buffon's needle," a long line segment dropped at 

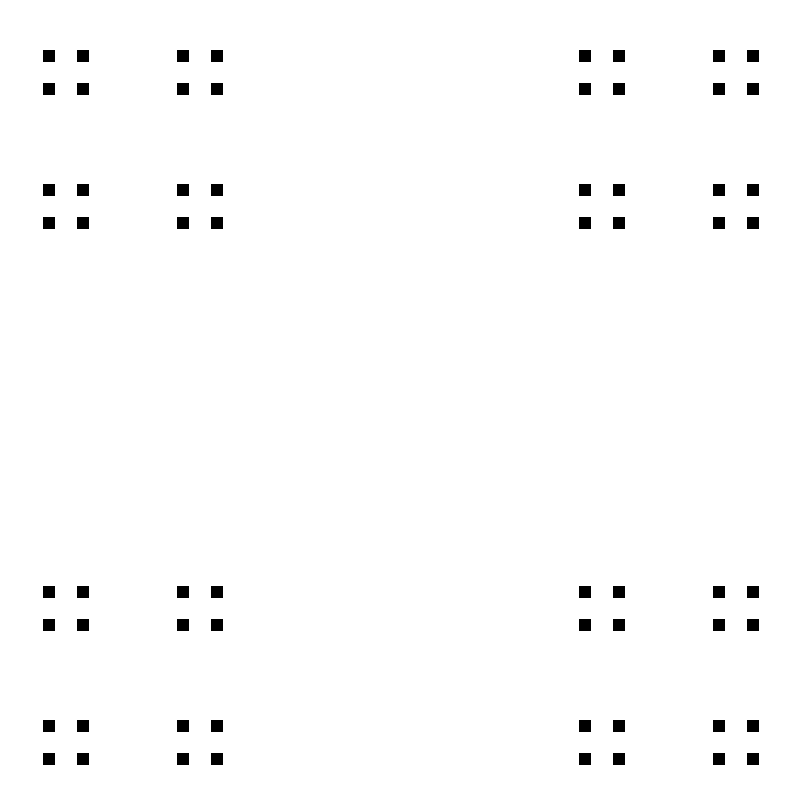

Figure 1. The Cantor set $K^{2}$, third stage of the construction.

random, hits $E$. (More precisely, suppose the needle's length is greater than $\sqrt{8}$, pick the distance $r$ from the origin to the needle uniformly in $[0, \sqrt{2}]$, and locate the center of the needle at a uniformly chosen point on the circle $\{|z|=r\}$.)

Now consider the $n$-th stage of the Cantor set construction for $K$,

$$
\begin{aligned}
& K_{n}=\left\{\sum_{k=1}^{\infty} a_{k} 4^{-k}: a_{k}\right. \\
& \qquad\{0,3\} \\
& \left.\qquad \text { for } 1 \leq k \leq n \text { and } a_{k} \in\{0,1,2,3\} \text { for } k>n\right\} .
\end{aligned}
$$

Then $K_{n}^{2}$ is a union of $4^{n}$ squares of side $4^{-n}$ (see Figure 1 for a picture of $\left.K_{3}^{2}\right)$. Clearly, $\operatorname{Fav}\left(K^{2}\right)=0$ implies $\lim _{n \rightarrow \infty} \operatorname{Fav}\left(K_{n}^{2}\right)=0$. We are interested in the behavior of $\operatorname{Fav}\left(K_{n}^{2}\right)$ as $n \rightarrow \infty$. A lower bound $\operatorname{Fav}\left(K_{n}^{2}\right) \geq \frac{c}{n}$ for some $c>0$ follows from Mattila $[\mathbf{1 4}, 1.4]$. Our main result is a quantitative 
upper bound. For $y \geq 1$ let

$$
\log _{*} y=\min \{n \geq 0: \underbrace{\log \log \ldots \log y \leq 1\} .}_{n}
$$

Theorem 1.1. There exist $C, a>0$ such that

$$
\operatorname{Fav}\left(K_{n}^{2}\right) \leq C \exp \left[-a \log _{*} n\right] \quad \text { for all } n \in \mathbb{N} \text {. }
$$

\section{Remarks.}

1. The convergence of the upper bound to zero is extremely slow, but it is the best we could get. It is still much better than a purely qualitative convergence statement. The lower bound $\frac{c}{n}$ seems closer to the truth. In Theorem 2.2, proved in Section 6, we analyze a random analog of the Cantor set $K^{2}$. We show that, with high probability, the Favard length of the $n$ th stage in the construction has upper and lower bounds that are constant multiples of $n^{-1}$.

2. For $\rho \leq 4^{-n}$, the $\rho$-neighborhood $K(\rho)=\{\mathbf{x}: \operatorname{dist}(\mathbf{x}, K) \leq \rho\}$ of $K$ can be covered by nine translates of $K_{n}$, so $\operatorname{Fav}(K(\rho)) \leq 9 \operatorname{Fav}\left(K_{n}\right)$.

3. It follows from the results of Kenyon [9] and Lagarias and Wang [10] that $\left|\operatorname{proj}_{\theta} K^{2}\right|=0$ for all $\theta$ such that $\tan \theta$ is irrational. However, this information does not seem to help obtain an upper bound for $\operatorname{Fav}\left(K_{n}^{2}\right)$.

4. The set $K^{2}$ was one of the first examples of sets of positive length and zero analytic capacity, see [3] for a survey. Recently Mateu, Tolsa and Verdera [12] proved that the analytic capacity of $K_{n}^{2}$ is bounded above and below by constant multiples of $n^{-1 / 2}$. The analytic capacity of certain related sets of non $\sigma$-finite length was determined by Mattila [16]. We consider the Favard length of such sets in Proposition 7.2.

In the next section we state our results for a class of planar self-similar Cantor sets. The method used for estimating the Favard length of the $n$-th stage of the construction also yields some information about gauges in which almost every projection of the Cantor set has zero Hausdorff measure. The proof of the main theorem for homogeneous self-similar sets (such as $K^{2}$ ) is presented in Sections 3 and 4. The non-homogeneous case, which is more involved, is dealt with in Section 5. Favard length of random Cantor sets is considered in Section 6. Section 7 contains some further extensions, remarks and unsolved problems. 


\section{Statement of results.}

Consider a self-similar set $\Lambda \subset \mathbb{R}^{2}$, defined as the unique nonempty compact satisfying

$$
\Lambda=\bigcup_{i=1}^{m} S_{i} \Lambda \text { where } S_{i}(\mathbf{x})=r_{i} \mathbf{x}+b_{i}, \text { with } r_{i} \in(0,1) \text { and } b_{i} \in \mathbb{R}^{2} .
$$

We assume that the Strong Separation Condition (SSC) holds, i.e., that $S_{i}(\Lambda) \cap S_{j}(\Lambda)=\emptyset$ for $i \neq j$. The similarity dimension is defined as the unique solution $s$ of the equation $\sum_{i=1}^{m} r_{i}^{s}=1$. It is well-known that the Strong Separation Condition, and even the weaker Open Set Condition, imply that the Hausdorff dimension $\operatorname{dim}_{H} \Lambda$ equals the similarity dimension $s$, and the $s$-dimensional Hausdorff measure $\mathcal{H}^{s}(\Lambda)$ is positive and finite.

First suppose that $s=1$. Then $\Lambda$ is an irregular 1 -set, and thus by Besicovitch's theorem (see [4, Theorem 6.13]) $\operatorname{Fav}(\Lambda)=0$. Let $\Lambda(\rho)=$ $\{\mathbf{x}: \operatorname{dist}(\mathbf{x}, \Lambda) \leq \rho\}$ denote the $\rho$-neighborhood of the set $\Lambda$. Clearly, $\lim _{\rho \rightarrow 0} \operatorname{Fav}(\Lambda(\rho))=0$. Mattila $[\mathbf{1 4}, 1.4]$ proved the lower bound

$$
\operatorname{Fav}(\Lambda(\rho)) \geq c\left(\log \left(\frac{1}{\rho}\right)\right)^{-1} \text { for all } \rho>0,
$$

for some $c>0$. (This lower bound follows from an energy estimate; it does not use self-similarity, but only positivity of $\mathcal{H}^{1}(\Lambda)$.) Our main result is the following upper bound.

Theorem 2.1. Assuming that the SSC holds and $s=1$, we have for some $C, a>0$

$$
\operatorname{Fav}(\Lambda(\rho)) \leq C \exp \left[-a \log _{*}\left(\frac{1}{\rho}\right)\right] \quad \text { for all } \rho>0 .
$$

Remark. The self-similar set is called homogeneous if $r_{i}=r$ for all $i \leq m$. The Cantor set $K^{2}$ in Section 1 is homogeneous. For a homogeneous set $\Lambda$, it is equivalent (up to uniform multiplicative constants) to consider the Favard length $\operatorname{Fav}\left(\Lambda_{n}\right)$ of the $n$th stage of the construction and $\operatorname{Fav}(\Lambda(\rho))$, with $\rho=r^{n}$.

We now consider random analogs of the sets $K_{n}^{2}$ from the introduction.

Partition the unit square into four dyadic subsquares of side $1 / 2$, and in each of these choose, uniformly at random, a dyadic subsquare of side $1 / 4$. Denote the union of four (closed) squares so obtained $\mathcal{R}_{1}$. Inductively, given $\mathcal{R}_{k}$ which is a union of $4^{k}$ dyadic squares of side $2^{-2 k}$, we partition each of them into four dyadic subsquares of side $2^{-2 k-1}$, and in each of these $4^{k+1}$ squares choose, uniformly at random, a dyadic subsquare of side $2^{-2 k-2}$, all these choices being independent. Call the union of $4^{k+1}$ (closed) squares so obtained $\mathcal{R}_{k+1}$. An example of $\mathcal{R}_{3}$ is given in Figure 2 . 

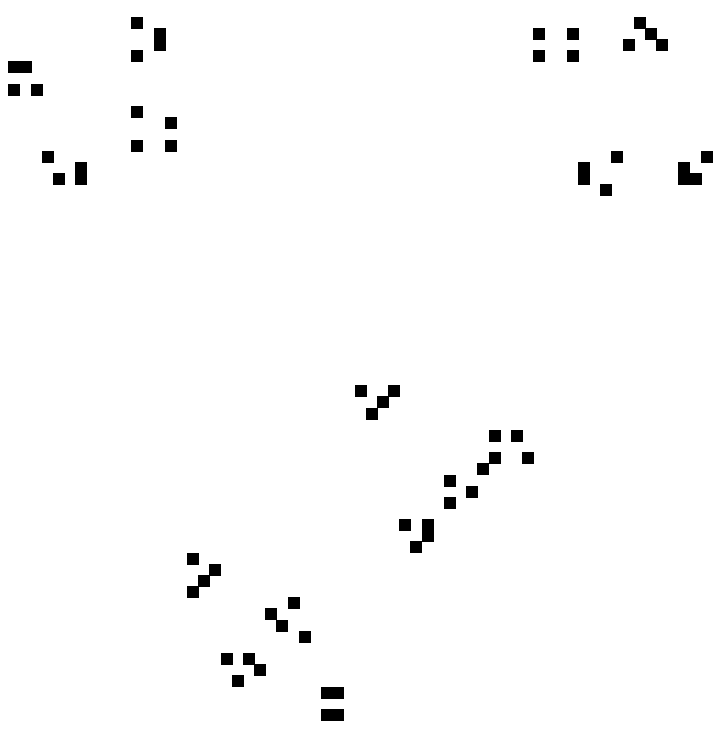

Figure 2. A random set $\mathcal{R}_{3}$.

Finally, write $\mathcal{R}=\bigcap_{k=1}^{\infty} \mathcal{R}_{k}$. Clearly $0<\mathcal{H}^{1}(\mathcal{R})<\infty$, and the arguments of Mattila $[\mathbf{1 4}, 1.4]$ still imply that $\operatorname{Fav}\left(\mathcal{R}_{n}\right) \geq \frac{c}{n}$. Denoting expectation by $\mathbf{E}$, we have:

\section{Theorem 2.2.}

$$
\mathbf{E}\left[\operatorname{Fav}\left(\mathcal{R}_{n}\right)\right] \leq \frac{C}{n}
$$

for some $C<\infty$. Consequently, with probability 1 ,

$$
\liminf _{n \rightarrow \infty} n \cdot \operatorname{Fav}\left(\mathcal{R}_{n}\right)<\infty .
$$

Next, we return to consider self-similar sets $\Lambda$ as in (2.1), but only assume that their similarity dimension satisfies $s \leq 1$. Let

$$
\mathcal{I} P(\Lambda)=\left\{\theta \in[0, \pi]:\left.\operatorname{proj}_{\theta}\right|_{\Lambda} \text { is not one-to-one }\right\}
$$

(the letters "I $P$ " stand for "intersection parameters"). It is easy to see that if $s=1$, then $\mathcal{I} P(\Lambda)=[0, \pi]$. (Indeed, if $\left.\operatorname{proj}_{\theta}\right|_{\Lambda}$ is one-to-one, then $\operatorname{proj}_{\theta}(\Lambda)$ is a self-similar set on the real line satisfying the Strong Separation 
Condition. Increasing the contraction rates $r_{i}$ slightly (maintaining strong separation), we would get a subset of $\mathbb{R}$ with Hausdorff dimension greater than 1 , a contradiction.)

It was proved in [18, Theorem 1.2] that if the set $\mathcal{I} P(\Lambda)$ contains a nonempty interval $J$, then $\mathcal{H}^{s}\left(\operatorname{proj}_{\theta} \Lambda\right)=0$ for a.e. $\theta \in J$. Here we exhibit an explicit gauge function $\phi(t)$ such that $\lim _{t \rightarrow 0} \frac{\phi(t)}{t^{s}}=\infty$ but $\mathcal{H}^{\phi}\left(\operatorname{proj}_{\theta} \Lambda\right)=0$ for a.e. $\theta \in J$.

Theorem 2.3. If the $\mathrm{SSC}$ holds, $s \leq 1$, and there is an interval $J \subset \mathcal{I} P(\Lambda)$, then $\mathcal{H}^{\phi}\left(\operatorname{proj}_{\theta} \Lambda\right)=0$ for a.e. $\theta \in J$, where

$$
\phi(t)=t^{s} \exp \left[L \log _{*}(1 / t)\right]
$$

with $L \in(0, \log 2)$.

Sufficient conditions for the existence of an interval $J \subset \mathcal{I} P(\Lambda)$ were found in [18]. For instance, Theorem 2.3 applies to the planar Cantor set $K^{(r)} \times K^{(r)}$ where $K^{(r)}=\left\{\sum_{n=1}^{\infty} a_{n} r^{n}: a_{n} \in\{0,1\}\right\}$, with $r \in\left(\frac{1}{6}, \frac{1}{4}\right)$. It is shown in $\left[\mathbf{1 8}\right.$, Example 6.1] that $J=\left[\arctan \frac{1-2 r}{r}, \arctan \frac{2}{1-3 r}\right] \subset \mathcal{I} P\left(K^{(r)}\right)$.

\section{Proof of Theorem 2.1 (the homogeneous case).}

Here we prove Theorem 2.1 in the case when $r_{i}=r$; this includes Theorem 1.1. Note that $s=1$ implies $r=m^{-1}$. Since some of the lemmas will also be used in the proof of Theorem 2.3, up to a point we allow any value of $s \leq 1$. The more technical proofs of lemmas are postponed until the next section.

Let $m \geq 2, \mathcal{A}=\{1, \ldots, m\}$ and $\mathcal{A}^{*}=\bigcup_{n>0} \mathcal{A}^{n}$. Write $|u|=n$ for $u \in \mathcal{A}^{n}$ and let $\left.\omega\right|_{n}=\omega_{1} \ldots \omega_{n}$ for $\omega \in \mathcal{A}^{*} \cup \mathcal{A}^{\mathbb{N}}$, with $|\omega| \geq n$. For $u \in \mathcal{A}^{n}$ we write $S_{u}=S_{u_{1}} \circ \ldots \circ S_{u_{n}}$ and $\Lambda_{u}=S_{u}(\Lambda)$. In our homogeneous case we have $S_{u}(\mathbf{x})=r^{n} \mathbf{x}+b_{u}$ for some $b_{u} \in \mathbb{R}^{2}$. It is convenient to identify the line through the origin with $\mathbb{R}$; formally we just let $\operatorname{proj}_{\theta}(x, y)=x \cos \theta+y \sin \theta$. For $\theta \in[0, \pi]$ and $u \in \mathcal{A}^{n}$ let

$$
S_{u}^{\theta}(x)=r^{n} x+b_{u}^{\theta}, x \in \mathbb{R}, \quad \text { where } b_{u}^{\theta}=\operatorname{proj}_{\theta} b_{u} .
$$

Observe that $\Lambda^{\theta}:=\operatorname{proj}_{\theta} \Lambda$ is a self-similar set on the real line satisfying $\Lambda^{\theta}=\bigcup_{i=1}^{m} S_{i}^{\theta}\left(\Lambda^{\theta}\right)$. The sets $\Lambda_{u}^{\theta}:=\operatorname{proj}_{\theta} \Lambda_{u}$ are called the cylinders of the self-similar set $\Lambda^{\theta}$. The map $\Pi_{\theta}: \mathcal{A}^{\mathbb{N}} \rightarrow \Lambda^{\theta}$ defined by

$$
\Pi_{\theta}(\omega)=\lim _{n \rightarrow \infty} S_{\left.\omega\right|_{n}}^{\theta}(0)=\sum_{n=1}^{\infty} r^{n-1} b_{\omega_{n}}^{\theta},
$$

is called the natural projection map. We equip the sequence space $\mathcal{A}^{\mathbb{N}}$ with the Bernoulli measure $\left(\frac{1}{m}, \ldots, \frac{1}{m}\right)^{\mathbb{N}}$. The projection of $\mu$, that is, $\nu_{\theta}:=$ $\mu \circ \Pi_{\theta}^{-1}$ is called the natural measure on $\Lambda^{\theta}$. 
Definition 3.1. Let $u, v$ be two words in $\mathcal{A}^{*}$ with $|u|=|v|=n$ and let $\theta \in[0, \pi]$. We say that $S_{u}^{\theta}$ and $S_{v}^{\theta}$ are $\varepsilon$-relatively close if

$$
\left|S_{u}^{\theta}(x)-S_{v}^{\theta}(x)\right| \leq \varepsilon r^{n} \quad \text { for all } x \in \Lambda^{\theta} .
$$

This definition is motivated by the work of Bandt and Graf [1]; it was recently used in [17]. In order to develop the setting needed for the proof of both Theorem 2.1 and Theorem 2.3, we fix a nonempty interval $J \subset \mathcal{I} P(\Lambda)$; if $s=1$ then we let $J=\mathcal{I} P(\Lambda)=[0, \pi]$.

Lemma 3.2. There exists $C_{1} \geq 1$ such that for all $\varepsilon \in(0,1]$ and all $n \in \mathbb{N}$, for any interval $I \subset J$, with $|I|=C_{1} r^{n}$, there is a subinterval $I^{\prime} \subset I$ satisfying:

(i) $\left|I^{\prime}\right| \geq C_{1}^{-2} \varepsilon|I|$ and

(ii) for every $\theta \in I^{\prime}$ there exist $u \neq v$ in $\mathcal{A}^{n}$ such that $S_{u}^{\theta}$ and $S_{v}^{\theta}$ are $\varepsilon$-relatively close.

This is a consequence of "transversality"; the proof is given in Section 4 .

Notation. Let $\Psi(n, k, \varepsilon)$ be the set of $\theta \in J$ such that there is no collection of distinct words $u_{1}, \ldots, u_{k}$, with $\left|u_{1}\right|=\ldots=\left|u_{k}\right| \leq n$, such that $S_{u_{j}}^{\theta}, j \leq k$, are pairwise $\varepsilon$-relatively close.

Lemma 3.3. There exist $c_{2}>0$ and $M>0$ such that

$$
|\Psi(n, 2, \varepsilon)| \leq M e^{-c_{2} n \varepsilon} \quad \text { for all } n \in N, \varepsilon \in(0,1] .
$$

This follows from Lemma 3.2; see Section 4 for the proof.

Lemma 3.4. If $n=\ell_{0}+j_{0}$, with $\ell_{0}, j_{0} \geq 1$, and $k \geq 2$, then

$$
\Psi(n, 2 k, \varepsilon) \subset \Psi\left(\ell_{0}, 2,(\varepsilon / 2) r^{j_{0}}\right) \cup \Psi\left(j_{0}, k,(\varepsilon / 2)\right) .
$$

Proof. Suppose that $\theta$ is not in the right-hand side of (3.3). Then there exist distinct $u_{1}, u_{2}$, with $\left|u_{i}\right| \leq \ell_{0}$, such that $S_{u_{1}}^{\theta}$ and $S_{u_{2}}^{\theta}$ are $\frac{\varepsilon}{2} r^{j_{0}}$-relatively close, and distinct $w_{1}, \ldots, w_{k}$, with $\left|w_{q}\right| \leq j_{0}$, such that $S_{w_{1}}^{\theta}, \ldots, S_{w_{k}}^{\theta}$ are pairwise $\frac{\varepsilon}{2}$-relatively close. Let $1 \leq p<q \leq k$. By self-similarity, $S_{u_{i} w_{p}}^{\theta}$ and $S_{u_{i} w_{q}}^{\theta}$ are $\frac{\varepsilon}{2}$-relatively close. Further, $S_{u_{1} w_{q}}^{\theta}$ and $S_{u_{2} w_{q}}^{\theta}$ are $\frac{\varepsilon}{2}$-relatively close, since $r^{j_{0}+\left|u_{i}\right|} \leq r^{\left|u_{i} w_{q}\right|}$, for $i=1,2$. This implies that $S_{u_{1} w_{p}}^{\theta}$ and $S_{u_{2} w_{q}}^{\theta}$ are $\varepsilon$-relatively close. Thus, we have found $2 k$ distinct words $u_{i} w_{q}$, with $i=1,2$ and $q \leq k$, of length $\leq n$, such that $S_{u_{i} w_{q}}^{\theta}$ are pairwise $\varepsilon$-relatively close, hence $\theta \notin \Psi(n, 2 k, \varepsilon)$.

Below we denote by $\log ^{i}$ and $\exp ^{i}$ the $i$-th iterate of log and exp respectively, assuming that $\log ^{0}$ is the identity map.

Lemma 3.5. There exists $c_{3}>0$ such that for all $i \geq 1$,

$$
\left|\Psi\left(n, 2^{i}, \varepsilon\right)\right| \leq M 2^{i-1} \exp \left[-c_{3} e^{-(i-1)}\left(\log ^{i-1} n\right) \varepsilon\right] \text { for all } n \in \mathbb{N}, \varepsilon \in(0,1]
$$


This is proved by induction, using Lemmas 3.3 and 3.4. See Section 4 for details. Now let

$$
n_{k}=\exp ^{k-1}\left(c_{3}^{-1} k e^{k-1}\right),
$$

so that, in view of (3.4),

$$
\left|\Psi\left(n_{k}, 2^{k}, 1\right)\right| \leq M 2^{k-1} e^{-k} .
$$

For $v, w \in \mathcal{A}^{*}$ we write $v \sqsubset w$ if $v$ is a subword of $w$, more precisely, if $w=v^{\prime} v v^{\prime \prime}$ where $v^{\prime}$ and/or $v^{\prime \prime}$ may be empty. Let

$$
N(k):=m^{n_{k}} \cdot n_{k} \cdot k .
$$

For $u_{1} \in \mathcal{A}^{*}$, with $\left|u_{1}\right| \leq n_{k}$, we have

$$
\begin{aligned}
\#\left\{u \in \mathcal{A}^{N(k)}: u_{1} \not \subset u\right\} & \leq\left(m^{n_{k}}-1\right)^{N(k) / n_{k}} \\
& =m^{N(k)}\left(1-m^{-n_{k}}\right)^{m^{n_{k} \cdot k}} \leq m^{N(k)} e^{-k} .
\end{aligned}
$$

Lemma 3.6. For any $\xi>0$ there exists $C_{\xi}>0$ such that

$$
\log _{*}\left(r^{-N(k)}\right) \leq C_{\xi}+(1+\xi) k .
$$

This is elementary; see Section 4 for a proof.

Proof of Theorem 2.1 (homogeneous case). Recall that now $s=1$, so $r=$ $m^{-1}$. We are going to show that, for some $c>0$ and $\gamma \in(0,1)$,

$$
\operatorname{Fav}(\Lambda(\rho)) \leq c \gamma^{k}, \quad \text { where } \rho=r^{N(k)} .
$$

By Lemma 3.6, this will imply (2.3).

Turning to the proof of (3.9), we note that by (3.6),

$$
\int_{\Psi\left(n_{k}, 2^{k}, 1\right)}\left|\operatorname{proj}_{\theta} \Lambda(\rho)\right| d \theta \leq M 2^{k-1} e^{-k}(\operatorname{diam}(\Lambda)+2) .
$$

Thus, it suffices to estimate $\left|\operatorname{proj}_{\theta} \Lambda(\rho)\right|$ from above for $\theta \notin \Psi\left(n_{k}, 2^{k}, 1\right)$.

Fix such a $\theta$ for the rest of the proof. By definition, this means that there exist words $u_{1}, \ldots, u_{2^{k}}$, each of length not greater than $n_{k}$, such that $S_{u_{j}}^{\theta}, j \leq 2^{k}$, are pairwise 1-relatively close. We have

$$
\operatorname{proj}_{\theta} \Lambda(\rho) \subset \bigcup_{|u|=N(k)} \Lambda_{u}^{\theta}(\rho)=\bigcup_{\substack{|u|=N(k) \\
u_{1} \not \subset u}} \Lambda_{u}^{\theta}(\rho) \cup \underset{\begin{array}{c}
|u|=N(k) \\
u_{1} \sqsubset u
\end{array}}{\bigcup} \Lambda_{u}^{\theta}(\rho)=: F_{1} \cup F_{2} .
$$

Since $|u|=N(k)$ we have

$$
\operatorname{diam}\left(\Lambda_{u}^{\theta}(\rho)\right)=\operatorname{diam}\left(\Lambda_{u}^{\theta}\right)+2 \rho \leq(2+\operatorname{diam}(\Lambda)) m^{-N(k)},
$$

hence, in view of (3.8),

$$
\left|F_{1}\right| \leq m^{N(k)} e^{-k}(2+\operatorname{diam}(\Lambda)) m^{-N(k)}=(2+\operatorname{diam}(\Lambda)) e^{-k} .
$$

It remains to estimate $\left|F_{2}\right|$. Suppose that $x \in F_{2}$. Then $x \in \Lambda_{u}^{\theta}(\rho)$ for some $u$ containing $u_{1}$ as a subword. We have $u=v u_{1} w$ for some (possibly empty) 
words $v$ and $w$. Then clearly $x \in \Lambda_{v u_{1}}^{\theta}(\rho)$. Recall that $S_{u_{1}}^{\theta}, \ldots, S_{u_{2^{k}}}^{\theta}$ are pairwise-1 close, hence $S_{v u_{1}}^{\theta}, \ldots, S_{v u_{2^{k}}}^{\theta}$ are pairwise-1 close as well. Let $q=$ $|v|+\left|u_{1}\right|$. Of course, $q \leq N(k)$. It follows that the ball $B(x):=B\left(x, c_{4} r^{q}\right)$, with $c_{4}=2+\operatorname{diam}(\Lambda)$, contains all $\Lambda_{v u_{j}}^{\theta}$, for $j \leq 2^{k}$. Therefore, the natural measure $\nu_{\theta}$ of the ball satisfies

$$
\nu_{\theta} B(x) \geq 2^{k} m^{-q}=2^{k-1} c_{4}^{-1}|B(x)| .
$$

By a classical covering theorem (see [15, Theorem 2.1]), we can choose a disjoint family $\left\{B_{j}\right\}$ of the balls $\left\{B(x): x \in F_{2}\right\}$ so that $F_{2} \subset \bigcup_{j} 5 B_{j}$. Thus,

$$
\left|F_{2}\right| \leq 5 \sum_{j}\left|B_{j}\right| \leq 5 c_{4} 2^{-(k-1)} \sum_{j} \nu_{\theta} B_{j} \leq 5 c_{4} 2^{-(k-1)},
$$

since $\nu_{\theta}$ is a probability measure. Combining this estimate with (3.10) and (3.11) yields (3.9), with $\gamma=2 / e$, and the proof is complete.

\section{Proof of the lemmas.}

Proof of Lemma 3.2. This is an easy "transversality argument", essentially contained in the proof of [18, Theorem 2.1(i)]. We provide a proof for the reader's convenience.

By increasing $C_{1}$ we can assume that $n$ is sufficiently large. Let $\theta_{0} \in$ $\mathcal{I} P(\Lambda)$. This means, by definition, that $\left.\operatorname{proj}_{\theta_{0}}\right|_{\Lambda}$ is not one-to-one, hence there exist $i \neq j$ such that $\Lambda_{i}^{\theta_{0}} \cap \Lambda_{j}^{\theta_{0}} \neq \emptyset$. Fix $\varepsilon>0$ and $n \in \mathbb{N}$. There exist $u, v \in \mathcal{A}^{n}$ such that $u_{1}=i, v_{1}=j$, and $\Lambda_{u}^{\theta_{0}} \cap \Lambda_{v}^{\theta_{0}} \neq \emptyset$.

Recall that $S_{u}(\mathbf{x})=r^{n} \mathbf{x}+b_{u}$, where $\mathbf{x}, b_{u} \in \mathbb{R}^{2}$, and $S_{u}^{\theta}(x)=r^{n} x+b_{u}^{\theta}$. Consider the function $f(\theta)=b_{u}^{\theta}-b_{v}^{\theta}=\left(b_{u}-b_{v}\right) \cdot(\cos \theta, \sin \theta)$. Observe that $S_{u}^{\theta}$ and $S_{v}^{\theta}$ are $\varepsilon$-relatively close if and only if $|f(\theta)| \leq \varepsilon r^{n}$. We have $|f(\theta)|^{2}+\left|f^{\prime}(\theta)\right|^{2}=\left|b_{u}-b_{v}\right|^{2}$. Thus,

$$
\eta^{2}-|f(\theta)|^{2} \leq\left|f^{\prime}(\theta)\right|^{2} \leq(\operatorname{diam}(\Lambda))^{2} \quad \text { for } \theta \in[0, \pi],
$$

where $\eta=\min \left\{\operatorname{dist}\left(\Lambda_{p}, \Lambda_{q}\right): 1 \leq p<q \leq m\right\}$. Note that $\eta>0$ by the Strong Separation Condition. Since $\Lambda_{u}^{\theta_{0}} \cap \Lambda_{v}^{\theta_{0}} \neq \emptyset$, we have $\left|f\left(\theta_{0}\right)\right| \leq$ $\operatorname{diam}\left(\Lambda_{u}^{\theta_{0}}\right) \leq \operatorname{diam}(\Lambda) r^{n}$, which can be assumed less than $\frac{\eta}{2}$, since $n$ is large. Then $\left|f^{\prime}\left(\theta_{0}\right)\right| \geq \frac{\sqrt{3} \eta}{2}>\frac{\eta}{2}$ and it follows from (4.1) that there exists $\theta_{1}$, with $\left|\theta_{1}-\theta_{0}\right| \leq \frac{2}{\eta} \operatorname{diam}(\Lambda) r^{n}$, such that $f\left(\theta_{1}\right)=0$. Then $S_{u}^{\theta_{1}} \equiv S_{v}^{\theta_{1}}$, and for all $\theta \in$ $\left(\theta_{1}-\frac{\varepsilon}{\operatorname{diam}(\Lambda)} r^{n}, \theta_{1}+\frac{\varepsilon}{\operatorname{diam}(\Lambda)} r^{n}\right)$, by (4.1), the maps $S_{u}^{\theta}$ and $S_{v}^{\theta}$ are $\varepsilon$-relatively close. This implies that the interval $\left[\theta_{0}-\frac{2}{\eta} \operatorname{diam}(\Lambda) r^{n}, \theta_{0}+\frac{2}{\eta} \operatorname{diam}(\Lambda) r^{n}\right]$ contains a subinterval $I^{\prime}$ of length $\min \left\{\frac{\varepsilon}{\operatorname{diam}(\Lambda)}, \frac{4 \operatorname{diam}(\Lambda)}{\eta}\right\} r^{n}$ which has the property (ii) from the statement of the lemma. The claim for an arbitrary interval $I \subset J$ now follows easily. 
Proof of Lemma 3.3. This proof is analogous to that of [19, Lemma 4.1].

Fix $\ell \in \mathbb{N}$ so that $r^{\ell} \leq \frac{1}{2}(1-r)$ and $\ell_{0}$ such that $C_{1} r^{\ell_{0}} \leq|J|$. We are going to construct inductively a family of compact sets $F_{0} \supset F_{1} \supset \cdots \supset F_{n}$, such that $\left|F_{n}\right| \leq e^{-c n \varepsilon}$ for some $c>0$ and $F_{n}$ is a union of $2^{n}$ intervals, each of length at least $C_{1} r^{\ell_{0}+\ell n}$. Most importantly, we will have that $F_{n} \supset$ $\Psi\left(\ell_{0}+\ell n, 2, \varepsilon\right)$. (Observe that $\Psi(k, 2, \varepsilon)$ are nested, decreasing with $k$, by the definition of these sets, so the desired estimate will follow.)

We can take $F_{0}=J$. Suppose that we already have $F_{n}$, for some $n \geq 0$, and we need to construct $F_{n+1}$. Let $I$ be any of the $2^{n}$ intervals of $F_{n}$ and find $k \leq n$ so that $C_{1} r^{k} \leq|I|<C_{1} r^{k-1}$. By assumption, $k \leq \ell_{0}+\ell n$. Let $I^{\prime}$ be the subinterval of $I$ of length $C_{1} r^{k+1}$ with the same center. By Lemma 3.2 , there is a subinterval $I^{\prime \prime} \subset I^{\prime}$ of length $\geq C_{1}^{-1} \varepsilon r^{k+1}$, which misses $\Psi(k+1,2, \varepsilon) \supset \Psi\left(\ell_{0}+\ell(n+1), 2, \varepsilon\right)$. Removing the interior of $I^{\prime \prime}$ makes two closed intervals out of $I$, each of length at least $\frac{1}{2} C_{1}\left(r^{k}-r^{k+1}\right) \geq$ $C_{1} r^{k+\ell} \geq C_{1} r^{\ell_{0}+\ell(n+1)}$. In this way we construct $F_{n+1}$, a union of $2^{n+1}$ intervals. It remains to observe that

$$
\left|I \backslash I^{\prime \prime}\right| \leq|I|-C_{1}^{-1} \varepsilon r^{k+1} \leq|I|\left(1-C_{1}^{-2} \varepsilon\right) .
$$

Thus, $\left|F_{n+1}\right| \leq\left(1-C_{1}^{-2} \varepsilon\right)\left|F_{n}\right| \leq e^{-C_{1}^{2} \varepsilon}\left|F_{n}\right|$, and the desired statement follows.

Proof of Lemma 3.5. We are going to prove (3.4) by induction in $i$. We can assume that $c_{3} \leq c_{2}$; then the case $i=1$ is just (3.2). Further, we can assume that $n \geq N_{0}$ and $\log ^{i-1} n \geq M_{0}$ for any fixed constants $N_{0}, M_{0}$, since otherwise (3.4) holds trivially for $c_{3}>0$ sufficiently small.

Suppose that (3.4) holds for some $i \geq 1$. Then by (3.3) and (3.2),

$$
\begin{aligned}
& \left|\Psi\left(n, 2^{i+1}, \varepsilon\right)\right| \\
& \leq\left|\Psi\left(\ell_{0}, 2,(\varepsilon / 2) r^{j_{0}}\right)\right|+\left|\Psi\left(j_{0}, 2^{i},(\varepsilon / 2)\right)\right| \\
& \leq M \exp \left[-c_{2} \ell_{0}(\varepsilon / 2) r^{j_{0}}\right]+M 2^{i-1} \exp \left[-c_{3} e^{-(i-1)}\left(\log ^{i-1} j_{0}\right)(\varepsilon / 2)\right] \\
& =: M\left(A_{1}+A_{2}\right),
\end{aligned}
$$

where $n=\ell_{0}+j_{0}$. Let $j_{0}$ be the smallest integer $\geq \frac{1}{2} \frac{\log n}{|\log r|}$. Then we have for $n$ sufficiently large:

$$
\begin{gathered}
\ell_{0}=n-j_{0} \geq n-\frac{1}{2} \frac{\log n}{|\log r|}-1 \geq \frac{n}{2}, \\
r^{j_{0}} \geq r^{\frac{1}{2} \frac{\log n}{|\log r|}-1}=r^{-1} n^{-1 / 2},
\end{gathered}
$$

hence

$$
A_{1} \leq \exp \left[-c_{2}(n / 2) r^{-1} n^{-1 / 2}(\varepsilon / 2)\right]=\exp \left[-c_{2}(4 r)^{-1} n^{1 / 2} \varepsilon\right]
$$


Let $\alpha:=2|\log r|$. Note that $\alpha>1$ since $r \leq m^{-1} \leq \frac{1}{2}$. Turning to $A_{2}$ in (4.2), we obtain from our choice of $j_{0}$ :

$$
A_{2} \leq 2^{i-1} \exp \left[-c_{3} e^{-(i-1)} \log ^{i-1}\left(\alpha^{-1} \log n\right) \cdot(\varepsilon / 2)\right] .
$$

If $i=1$, then in (4.2) we could use (3.2) for the second summand as well, in which case

$$
A_{2} \leq 2^{i-1} \exp \left[-c_{2} \alpha^{-1} \log n \cdot(\varepsilon / 2)\right] \leq 2^{i-1} \exp \left[-c_{3} \log n \cdot(\varepsilon / 2)\right],
$$

assuming $c_{3} \leq c_{2} \alpha^{-1}$. For $i \geq 2$ we use the elementary inequality

$$
\log (x+y) \leq \log x+y \quad \text { for all } x \geq 1, y \geq 0 .
$$

We can assume that $\log ^{i-1} n \geq \log \alpha+1$, since otherwise (3.4) holds trivially for $c_{3}>0$ sufficiently small. Then applying (4.6) $i-2$ times to $\log ^{2} n=$ $\log \left(\alpha^{-1} \log n\right)+\log \alpha$ we obtain

$$
\log ^{i} n \leq \log ^{i-1}\left(\alpha^{-1} \log n\right)+\log \alpha .
$$

Combining this with (4.4) and (4.5) yields

$$
A_{2} \leq 2^{i-1} \exp \left[-c_{3} e^{-(i-1)}\left(\log ^{i} n-\log \alpha\right)(\varepsilon / 2)\right] \text { for all } i \geq 1 .
$$

In view of (4.3), the induction step will be finished once we check the inequality

$$
\begin{aligned}
& \exp \left[-c_{2}(4 r)^{-1} n^{1 / 2} \varepsilon\right]+2^{i-1} \exp \left[-c_{3} e^{-(i-1)}\left(\log ^{i} n-\log \alpha\right)(\varepsilon / 2)\right] \\
& \leq 2^{i} \exp \left[-c_{3} e^{-i}\left(\log ^{i} n\right) \varepsilon\right] .
\end{aligned}
$$

This is equivalent to

$$
\begin{aligned}
1 \geq & 2^{-i} \exp \left[\left(c_{3} e^{-i} \log ^{i} n-c_{2}(4 r)^{-1} n^{1 / 2}\right) \varepsilon\right] \\
& +2^{-1} \exp \left[c_{3} \varepsilon e^{-(i-1)}\left(\log ^{i} n \cdot\left(e^{-1}-2^{-1}\right)+2^{-1} \log \alpha\right)\right]=: B_{1}+B_{2} .
\end{aligned}
$$

We have

$$
c_{3} e^{-i} \log ^{i} n-c_{2}(4 r)^{-1} n^{1 / 2} \leq c_{2} \log n-c_{2}(4 r)^{-1} n^{1 / 2}<0
$$

for $n$ sufficiently large, hence $B_{1} \leq 2^{-i}$. Further, we can assume that

$$
\log ^{i} n>\log \alpha \cdot\left(1 / 2-e^{-1}\right)^{-1}
$$

then $B_{2} \leq \frac{1}{2}$. This implies (4.7), and the proof of the lemma is complete.

Proof of Lemma 3.6. It follows from (4.6) and (1.1) that

$$
\log _{*}(x+y) \leq \log _{*} x+\log _{*}(1+y) \quad \text { for all } x \geq 1, y \geq 0 .
$$


Using this inequality, (3.7), and (3.5), we obtain

$$
\begin{aligned}
\log _{*}\left(r^{-N(k)}\right) & \leq 1+\log _{*}\left(\log \left(r^{-1}\right)+\log N(k)\right) \\
& \leq \text { const }+\log _{*}\left(n_{k} \log m+\log n_{k}+\log k\right) \\
& \leq \text { const }+\log _{*} n_{k} \\
& =\text { const }+(k-1)+\log _{*}\left(c_{3}^{-1} k e^{k-1}\right) \\
& \leq \text { const }+k+\log _{*} k .
\end{aligned}
$$

Now the desired statement is immediate.

\section{Non-homogeneous case.}

Here we prove Theorem 2.1 in full generality and Theorem 2.3. The proofs follow the same path most of the way. We use the same notation as in Section 3, as much as possible, so the same letters often represent different but analogous objects here and there.

We have $S_{u}(\mathbf{x})=r_{u} \mathbf{x}+b_{u}$ for some $b_{u} \in \mathbb{R}^{2}$, where $r_{u}=r_{u_{1}} \cdot \ldots \cdot r_{u_{n}}$. The natural projection map onto $\Lambda^{\theta}$ is defined by $\Pi_{\theta}(\omega)=\lim _{n \rightarrow \infty} S_{\left.\omega\right|_{n}}^{\theta}(0)$. The natural measure on $\Lambda^{\theta}$ is $\nu_{\theta}=\mu \circ \Pi_{\theta}^{-1}$, where $\mu=\left(r_{1}^{s}, \ldots, r_{m}^{s}\right)^{\mathbb{N}}$ and $s \leq 1$ is the similarity dimension of $\Lambda$. For $\delta>0$ consider the "cut-set" $\mathcal{W}(\delta)=\left\{u \in \mathcal{A}^{*}: r_{u} \leq \delta, r_{u^{\prime}}>\delta\right\}$ where $u^{\prime}$ is obtained from $u$ by dropping the last symbol. Let $r_{\min }=\min \left\{r_{i}: i \leq m\right\}$ and $r_{\max }=\max \left\{r_{i}: i \leq m\right\}$. For $u, v \in \mathcal{W}(\delta)$ we have $r_{\min } \leq r_{u} / r_{v} \leq r_{\min }^{-1}$. Throughout this section we fix a nonempty interval $J \subset \mathcal{I} P(\Lambda)$ (assuming that it exists). Let $X_{\Lambda}=$ $\left[-d_{\Lambda}, d_{\Lambda}\right]$ where $d_{\Lambda}=\max \{|\mathbf{x}|: \mathbf{x} \in \Lambda\}$. Observe that $S_{i}^{\theta}\left(X_{\Lambda}\right) \subset X_{\Lambda}$ for all $i \leq m$ and all $\theta \in[0, \pi]$.

Definition 5.1. Let $\theta \in[0, \pi]$ and $u, v \in \mathcal{A}^{*}$. We say that $S_{u}^{\theta}$ and $S_{v}^{\theta}$ are $\varepsilon$-relatively close at $x$ if

$$
\left|S_{u}^{\theta}(x)-S_{v}^{\theta}(x)\right| \leq \varepsilon \min \left\{r_{u}, r_{v}\right\} .
$$

Lemma 5.2. There exists $C_{1} \geq 1$ such that for all $x \in X_{\Lambda}$, for all $\varepsilon \in(0,1]$ and all $\delta>0$, for any interval $I \subset J$, with $|I|=C_{1} \delta$, there is a subinterval $I^{\prime} \subset I$ such that $\left|I^{\prime}\right| \geq C_{1}^{-2} \varepsilon|I|$ and for every $\theta \in I^{\prime}$ there exist $u \neq v$ in $\mathcal{W}(\delta)$ such that $S_{u}^{\theta}$ and $S_{v}^{\theta}$ are $\varepsilon$-relatively close at $x$.

Proof. The proof is analogous to the proof of Lemma 3.2. Let $g(\theta)=$ $S_{u}^{\theta}(x)-S_{v}^{\theta}(x)=\left(r_{u}-r_{v}\right) x+f(\theta)$ where $f(\theta)=b_{u}^{\theta}-b_{v}^{\theta}$. If $\theta_{0} \in \mathcal{I} P(K)$, then $\left|g\left(\theta_{0}\right)\right| \leq 4 \delta d_{\Lambda}$ which can be assumed small, increasing $C_{1}$ if necessary. Since $g^{\prime}(\theta)=f^{\prime}(\theta)$, the rest of the proof of Lemma 3.2 transfers.

Notation. For $x \in X_{\Lambda}, k \geq 2, \varepsilon \in(0,1]$ and $n \geq 1$ denote by $\Phi(n, k, x, \varepsilon)$ the set of $\theta \in J$ such that there is no collection of distinct words $u_{1}, \ldots, u_{k}$, with $r_{u_{j}} \geq r_{\max }^{n}$, such that $S_{u_{j}}^{\theta}, j \leq k$, are pairwise $\varepsilon$-relatively close at $x$. Denote by $\Phi^{\prime}(n, k, x, \varepsilon)$ the analogous set where it is required, in addition, 
that $r_{\min } \leq r_{u_{i}} / r_{u_{j}} \leq r_{\min }^{-1}$ for $i, j \leq k$. By the definition, $\Phi^{\prime}(n, k, x, \varepsilon) \supset$ $\Phi(n, k, x, \varepsilon)$. Further, let

$$
\Psi(n, k, \varepsilon)=\bigcup_{x \in X_{\Lambda}} \Phi(n, k, x, \varepsilon) \text { and } \Psi^{\prime}(n, k, \varepsilon)=\bigcup_{x \in X_{\Lambda}} \Phi^{\prime}(n, k, x, \varepsilon) .
$$

Lemma 5.3. There exist $c_{2}>0$ and $M>0$ such that

$$
\left|\Psi^{\prime}(n, 2, \varepsilon)\right| \leq M \varepsilon^{-1} e^{-c_{2} n \varepsilon} \quad \text { for all } n \in N, \varepsilon \in(0,1] .
$$

Proof. Using Lemma 5.2 and repeating the proof of Lemma 3.3, we obtain for any fixed $x \in X_{\Lambda}$ that

$$
\left|\Phi^{\prime}(n, 2, x, \varepsilon)\right| \leq \widetilde{M} e^{-\widetilde{c} 1 n \varepsilon}
$$

for some constants $\widetilde{M}, \widetilde{c}_{1}>0$. Observe that if $S_{u}^{\theta}$ and $S_{v}^{\theta}$ are $\frac{\varepsilon}{2}$-relatively close at $x$ and $C^{-1} \leq r_{u} / r_{v} \leq C$, then $S_{u}^{\theta}$ and $S_{v}^{\theta}$ are $\varepsilon$-relatively close at $x^{\prime}$, provided that $\left|x^{\prime}-x\right| \leq \frac{\varepsilon}{2 C}$. Taking $C=r_{\min }^{-1}$ and choosing an $\frac{\varepsilon}{2 C}$-net $\mathcal{N}$ of $X_{\Lambda}$, we obtain

$$
\Psi^{\prime}(n, 2, \varepsilon) \subset \bigcup_{x \in \mathcal{N}} \Phi^{\prime}(n, 2, x, \varepsilon / 2) .
$$

Since $\#(\mathcal{N}) \leq$ const $\cdot \varepsilon^{-1}$, this and (5.2) yield (5.1).

Lemma 5.4. There exists $C \geq 1$ such that for $n=\ell_{0}+j_{0}$, with $\ell_{0}, j_{0} \geq 1$, and $k \geq 2$, we have

$$
\Psi(n, 2 k, \varepsilon) \subset \Psi\left(j_{0}, k, C^{-1} \varepsilon\right) \cup \Psi^{\prime}\left(\ell_{0}, 2, C^{-1} r_{\max }^{j_{0}} \varepsilon\right) .
$$

Proof. Fix $x_{0} \in X_{\Lambda}$. We want to show that $\Phi\left(n, 2 k, x_{0}, \varepsilon\right)$ lies in the righthand side of (5.3). Suppose that $\theta$ is not in the right-hand of (5.3). Then there exist distinct words $w_{1}, \ldots, w_{k}$, with $r_{w_{i}} \geq r_{\max }^{j_{0}}$, such that $S_{w_{i}}^{\theta}$ are pairwise $C^{-1} \varepsilon$-relatively close at $x_{0}$. Without loss of generality, suppose that $r_{w_{1}}=\min _{i \leq k} r_{w_{i}}$. Further, $\theta \notin \Psi^{\prime}\left(\ell_{0}, 2, C^{-1} r_{\max }^{j_{0}} \varepsilon\right)=\bigcup_{x \in X} \Phi^{\prime}\left(\ell_{0}, 2, x\right.$, $\left.C^{-1} r_{\max }^{j_{0}} \varepsilon\right)$, so there exist distinct $u_{1}, u_{2}$ such that $r_{u_{i}} \geq r_{\max }^{\ell_{0}}, r_{\min } \leq r_{u_{1}} / r_{u_{2}}$ $\leq r_{\min }^{-1}$, and $S_{u_{1}}^{\theta}$ and $S_{u_{2}}^{\theta}$ are $C^{-1} r_{\max }^{j_{0}} \varepsilon$-relatively close at $S_{w_{1}}^{\theta}\left(x_{0}\right) \in X_{\Lambda}$. Then $u_{i} w_{j}$, for $i=1,2$ and $j \leq k$, are all distinct and satisfy $r_{u_{i} w_{j}} \geq r_{\max }^{\ell_{0}+j_{0}}=r_{\max }^{n}$. We claim that $S_{u_{i} w_{j}}^{\theta}$ are pairwise $\varepsilon$-close at $x_{0}$ if $C$ is sufficiently large. This will imply that $\theta \notin \Phi\left(n, 2 k, x_{0}, \varepsilon\right)$, and since $x_{0}$ is arbitrary, the lemma will be proved.

We have for $i=1,2$ and for all $j \leq k$,

$$
\left|S_{u_{i} w_{1}}^{\theta}\left(x_{0}\right)-S_{u_{i} w_{j}}^{\theta}\left(x_{0}\right)\right| \leq r_{u_{i}} C^{-1} \varepsilon \min \left\{r_{w_{1}}, r_{w_{j}}\right\}=C^{-1} \varepsilon r_{u_{i} w_{1}} .
$$

Further,

$\left|S_{u_{1} w_{1}}^{\theta}\left(x_{0}\right)-S_{u_{2} w_{1}}^{\theta}\left(x_{0}\right)\right| \leq C^{-1} r_{\max }^{j_{0}} \varepsilon \min \left\{r_{u_{1}}, r_{u_{2}}\right\} \leq C^{-1} \varepsilon \min \left\{r_{u_{1} w_{1}}, r_{u_{2} w_{1}}\right\}$. 
Therefore, for $1 \leq p<q \leq k$,

$$
\begin{aligned}
\left|S_{u_{1} w_{p}}^{\theta}\left(x_{0}\right)-S_{u_{2} w_{q}}^{\theta}\left(x_{0}\right)\right| & \leq C^{-1} \varepsilon r_{w_{1}}\left(r_{u_{1}}+r_{u_{2}}+\min \left\{r_{u_{1}}, r_{u_{2}}\right\}\right) \\
& \leq C^{-1} \varepsilon r_{w_{1}}\left(2+r_{\min }^{-1}\right) \min \left\{r_{u_{1}}, r_{u_{2}}\right\} \\
& \leq C^{-1} \varepsilon\left(2+r_{\min }^{-1}\right) \min \left\{r_{u_{1} w_{p}}, r_{u_{2} w_{q}}\right\}
\end{aligned}
$$

and the claim follows with $C=2+r_{\min }^{-1}$. The lemma is proved.

Lemma 5.5. There exist $a>0$ and $b>1$ such that for all $\varepsilon \in(0,1], n \in \mathbb{N}$ and $i \geq 1$,

$$
\left|\Psi\left(n, 2^{i}, \varepsilon\right)\right| \leq M \varepsilon^{-1} b^{i} \exp \left[-a e^{-(i-1)}\left(\log ^{i-1} n\right) \varepsilon\right] .
$$

Proof is analogous to the proof of Lemma 3.5, based on Lemmas 5.3 and 5.4. We leave the details to the reader.

Let

$$
n_{k}=\exp ^{k-1}\left((\log b+1) a^{-1} k e^{k-1}\right),
$$

so that, in view of (5.4),

$$
\left|\Psi\left(n_{k}, 2^{k}, 1\right)\right| \leq M e^{-k} .
$$

Let

$$
N(k)=n_{k} \cdot\left\lceil r_{\min }^{-s}\right\rceil^{n_{k}} \cdot k .
$$

Similarly to the proof of Lemma 3.6, we deduce from (5.5) and (5.7) that

$$
\log _{*}\left(r_{\min }^{-N(k)}\right) \leq C_{\xi}+(1+\xi) k,
$$

for any $\xi>0$. For any $u_{1} \in \mathcal{A}^{*}$, with $\left|u_{1}\right| \leq n_{k}$, we have

$$
\sum_{\substack{|u|=N(k) \\ u_{1} \not \subset u}} r_{u}^{s} \leq\left(1-r_{\min }^{n_{k} s}\right)^{N(k) / n_{k}} \leq e^{-k} .
$$

Now suppose that $\theta \notin \Psi\left(n_{k}, 2^{k}, 1\right)$ and $x_{0} \in \Lambda^{\theta}$. Then $\theta \notin \Phi\left(n_{k}, 2^{k}, x_{0}, 1\right)$, so we can find distinct words $u_{1}, \ldots, u_{2^{k}}$, with $r_{u_{i}} \geq r_{\max }^{n_{k}}$, such that

$$
\left|S_{u_{i}}\left(x_{0}\right)-S_{u_{j}}\left(x_{0}\right)\right| \leq \min \left\{r_{u_{i}}, r_{u_{j}}\right\} \quad \text { for all } i, j \leq 2^{k} \text {. }
$$

Without loss of generality, assume that $r_{u_{1}}=\min \left\{r_{u_{i}}: i \leq 2^{k}\right\}$. We have

$$
\Lambda^{\theta}=\bigcup_{|u|=N(k)} \Lambda_{u}^{\theta}=\bigcup_{\begin{array}{c}
|u|=N(k) \\
u_{1} \not \subset u
\end{array}} \Lambda_{u}^{\theta} \cup \bigcup_{\begin{array}{c}
|u|=N(k) \\
u_{1} \sqsubset u
\end{array}} \Lambda_{u}^{\theta}=: Y_{u_{1}} \cup Z_{u_{1}} .
$$

We claim that for some $C \geq 1$,

$$
\forall x \in Z_{u_{1}}, \exists t \in\left[C^{-1} r_{\min }^{N(k)}, C r_{u_{1}}\right]: \nu_{\theta} B(x, t) \geq C^{-1} 2^{k} t^{s} .
$$

Indeed, suppose that $x \in \Lambda_{u}^{\theta}$ for some $u \in \mathcal{A}^{N(k)}$ such that $u_{1} \sqsubset u$. Then $u=v u_{1} w$ for some (possibly empty) words $v$ and $w$. Let $\omega \in \mathcal{A}^{\mathbb{N}}$ be such that $x_{0}=\Pi_{\theta}(\omega)$. For each $u_{j}$, with $2 \leq j \leq 2^{k}$, there exists a unique 
$q=q_{j} \in \mathbb{N} \cup\{0\}$ such that $\widetilde{u}_{j}:=\left.u_{j} \omega\right|_{q} \in \mathcal{W}\left(r_{u_{1}}\right)$. Notice that $S_{u_{j}}^{\theta}\left(x_{0}\right) \in \Lambda_{\widetilde{u}_{j}}^{\theta}$ whence $S_{v u_{j}}^{\theta}\left(x_{0}\right) \in \Lambda_{v \widetilde{u}_{j}}^{\theta}$. By (5.10), we have $\left|S_{v u_{j}}^{\theta}\left(x_{0}\right)-S_{v u_{1}}^{\theta}\left(x_{0}\right)\right| \leq r_{v u_{1}}$. Finally, $x \in \Lambda_{v u_{1}}^{\theta}$, which implies that the distance from $x$ to $\Lambda_{v \widetilde{u}_{j}}$ is at most $\operatorname{diam}\left(\Lambda_{v u_{1}}^{\theta}\right)+r_{v u_{1}}$. Since $r_{v \widetilde{u}_{j}}=r_{v} r_{\widetilde{u}_{j}} \leq r_{v u_{1}}$, we obtain that

$$
B\left(x, C^{\prime} r_{v u_{1}}\right) \supset \Lambda_{v u_{1}}^{\theta} \cup \bigcup_{j=2}^{2^{k}} \Lambda_{v \widetilde{u}_{j}}^{\theta}, \quad \text { where } C^{\prime}=1+2 \operatorname{diam}(\Lambda) .
$$

Therefore,

$$
\nu_{\theta} B\left(x, C^{\prime} r_{v u_{1}}\right) \geq r_{v u_{1}}^{s}+\sum_{j=2}^{2^{k}} r_{v \widetilde{u}_{j}}^{s} \geq 2^{k} r_{\min }^{-s} r_{v u_{1}}^{s} .
$$

This implies (5.12) since $r_{\min }^{N(k)} \leq r_{u} \leq r_{v u_{1}} \leq r_{u_{1}}$.

Proof of Theorem 2.1. Recall that now $s=1$. By (5.8), it suffices to show that for some $c>0$ and $\gamma \in(0,1)$, we have

$$
\operatorname{Fav}(\Lambda(\rho)) \leq c \gamma^{k}, \quad \text { where } \rho:=r_{\min }^{N(k)} .
$$

In view of (5.6), it is sufficient to estimate $\left|\Lambda^{\theta}(\rho)\right|$ from above for $\theta \notin$ $\Psi\left(n_{k}, 2^{k}, 1\right)$. Fix such a $\theta, x_{0} \in \Lambda^{\theta}$, and the words $u_{1}, \ldots, u_{2^{k}}$ as before, satisfying (5.10), and let $u_{1}$ be the word with the minimal $r_{u_{i}}$. By (5.11) we have $\Lambda^{\theta}(\rho)=Y_{u_{1}}(\rho) \cup Z_{u_{1}}(\rho)$. Clearly, $\operatorname{diam}\left(\Lambda_{u}^{\theta}(\rho)\right) \leq(2+\operatorname{diam}(\Lambda)) r_{u}$ for any $u \in \mathcal{A}^{N(k)}$, so (5.9), with $s=1$, implies that $\left|Y_{u_{1}}(\rho)\right| \leq$ const $\cdot e^{-k}$. Since $t \geq C^{-1} \rho$ in (5.12), the balls $B(x,(1+C) t)$, for $x \in Z_{u_{1}}$, cover the $\rho$ neighborhood $Z_{u_{1}}(\rho)$. Now (5.12) implies $\left|Z_{u_{1}}(\rho)\right| \leq$ const. $2^{-k}$, by repeating the argument at the end of Section 3 , and the proof is finished.

Proof of Theorem 2.3. We use the same setting as in the proof of Theorem 2.1, except that now $s \leq 1$ and $J \subset \mathcal{I} P(\Lambda)$ is a nonempty interval. In view of (5.6), the Borel-Cantelli Lemma implies that the set

$$
E:=\bigcup_{n=1}^{\infty} \bigcap_{k \geq n}\left(J \backslash \Psi\left(n_{k}, 2^{k}, 1\right)\right)
$$

has full Lebesgue measure in $J$. Thus, it is enough to show that $\mathcal{H}^{\phi}\left(\Lambda^{\theta}\right)=0$ for all $\theta \in E$.

Suppose that $\theta \in E$; then $\theta \in J \backslash \Psi\left(n_{k}, 2^{k}, 1\right)$ for all $k$ sufficiently large. We fix $x_{0} \in \Lambda^{\theta}$ and find $u_{1}=u_{1}(k)$ as above (now we have to make the dependence on $k$ explicit). For $\rho_{k}=r_{\min }^{N(k)}$ we have the decomposition (5.11) $\Lambda^{\theta}=Y_{u_{1}(k)} \cup Z_{u_{1}(k)}$. We can write $\Lambda^{\theta}=\Omega_{1} \cup \Omega_{2}$ where $\Omega_{1}$ is the set of $x$ which belong to infinitely many $Y_{u_{1}(k)}$ and $\Omega_{2}$ is the set of $x$ which 
belong to all $Z_{u_{1}(k)}$ for $k$ sufficiently large. Recall that $Y_{u_{1}(k)}=\bigcup_{\substack{|u|=N(k) \\ u_{1}(k) \not \subset u}} \Lambda_{u}^{\theta}$ and $\operatorname{diam}\left(\Lambda_{u}^{\theta}\right) \leq \operatorname{diam}(\Lambda) \cdot r_{u}$. Thus,

$$
\begin{aligned}
\mathcal{H}^{\phi}\left(\Omega_{1}\right) & \leq \text { const } \cdot \lim _{k \rightarrow \infty} \sum_{\substack{|u|=N(k) \\
u_{1}(k) \not \subset u}} \phi\left(r_{u}\right) \\
& =\text { const } \cdot \lim _{k \rightarrow \infty} \sum_{\substack{|u|=N(k) \\
u_{1}(k) \not \subset u}} r_{u}^{s} \exp \left[L \log _{*}\left(r_{u}^{-1}\right)\right] \\
& \leq \text { const } \cdot \lim _{k \rightarrow \infty} \sum_{\substack{|u|=N(k) \\
u_{1}(k) \not \subset u}} r_{u}^{s} \exp \left[L \log _{*}\left(\rho_{k}^{-1}\right)\right] \\
& \leq \text { const } \cdot \lim _{k \rightarrow \infty} e^{-k} e^{L(1+\xi) k}=0,
\end{aligned}
$$

using (5.9) and (5.8), with $0<\xi<L^{-1}-1$, in the last estimate. Recall that $L<\log 2<1$.

It remains to prove that $\mathcal{H}^{\phi}\left(\Omega_{2}\right)=0$. For any $x \in Z_{u_{1}(k)}$ we have by (5.12), with $t=t_{k} \geq C^{-1} \rho_{k}$,

$$
\begin{aligned}
\frac{\nu_{\theta} B\left(x, t_{k}\right)}{\phi\left(t_{k}\right)} & \geq \frac{\operatorname{const} \cdot 2^{k} t_{k}^{s}}{t_{k}^{s} \exp \left[L \log _{*}\left(2 t_{k}^{-1}\right)\right]} \\
& \geq \mathrm{const} \cdot 2^{k} \exp \left[-L \log _{*}\left(2 C \rho_{k}^{-1}\right)\right] \\
& \geq \mathrm{const} \cdot 2^{k} e^{-L(1+\xi) k} \rightarrow \infty, \text { as } k \rightarrow \infty .
\end{aligned}
$$

In the last line we used (5.8) with $0<\xi<L^{-1} \log 2-1$. Notice that $t_{k} \leq r_{u_{1}(k)} \rightarrow 0$, as $k \rightarrow \infty$ ( since $r_{u_{1}(k)}$ is the smallest among $r_{u_{i}(k)}, i \leq 2^{k}$, and all $u_{i}(k)$ are distinct). Thus, (5.15) implies

$$
\bar{D}_{\phi}\left(\nu_{\theta}, x\right):=\limsup _{t \rightarrow 0} \frac{\nu_{\theta} B(x, t)}{\phi(2 t)}=\infty \quad \text { for all } x \in \Omega_{2},
$$

and hence $\mathcal{H}^{\phi}\left(\Omega_{2}\right)=0$ by the Rogers-Taylor Density Theorem, see [20]. The proof of Theorem 2.3 is complete.

\section{Random Cantor sets.}

The proof of Theorem 2.2 is inspired by an argument of Lyons [11] involving percolation on trees; the negative dependence in the construction of $\mathcal{R}_{k}$ that arises from choosing exactly one of the four dyadic subsquares in the inductive step of the construction, makes the proof here a little more delicate.

Denote by $\mathcal{G}_{k}$ the collection of $4^{k}$ (closed) dyadic subsquares of the unit square $[0,1]^{2}$ having side length $2^{-k}$. We consider all dyadic subsquares as a rooted tree, with $[0,1]^{2}$ being the root and $\mathcal{G}_{k}$ being the set of nodes at the 
$k$ th level. For each node there are four edges leading to nodes at the next level, (its "children").

Let $\ell$ be a line intersecting $[0,1]^{2}$, that does not go through any of the vertices of the squares in $\mathcal{G}_{2 n}$. Further, let

$$
A_{n}(\ell)=\#\left\{B \in \mathcal{G}_{2 n}: B \cap \ell \neq \emptyset\right\} .
$$

Observe that

$$
A_{n}(\ell) \leq 2^{2 n+1} .
$$

To verify this we may assume, using symmetry, that $\ell$ forms an angle $\alpha \in$ $[0, \pi / 4]$ with the horizontal. Then $\ell$ intersects at most two squares in each of the $2^{2 n}$ columns of $\mathcal{G}_{2 n}$, and (6.1) follows.

Below $\mathbf{P}(E)$ denotes the probability of an event $E$.

Lemma 6.1. Suppose that the line $\ell$ does not hit any vertices of the squares in $\mathcal{G}_{2 n}$. Then

$$
\mathbf{P}\left(\mathcal{R}_{n} \cap \ell \neq \emptyset\right) \leq \frac{C_{1}}{n}
$$

for some constant $C_{1}>0$ independent of $\ell$ and $n$.

Proof of Theorem 2.2 assuming Lemma 6.1. Let $\theta \in[0, \pi]$ be such that the line $y \cos \theta=x \sin \theta$ is orthogonal to $\ell$, and let $\mathbf{n}$ be the unit normal vector for $\ell$. Then by Fubini's Theorem and Lemma 6.1,

$$
\mathbf{E}\left[\left|\operatorname{proj}_{\theta} \mathcal{R}_{n}\right|\right]=\int_{\mathbb{R}} \mathbf{P}\left(\mathcal{R}_{n} \cap(\ell+t \mathbf{n}) \neq \emptyset\right) d t \leq \sqrt{2} \frac{C_{1}}{n},
$$

and (2.4) follows by integrating over $\theta$.

Finally, (2.5) follows directly from (2.4) by Fatou's lemma.

Proof of Lemma 6.1. We label the four dyadic subsquares of a square as in Figure 3.

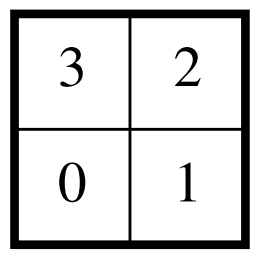

Figure 3. Labeling subsquares.

This labeling induces a natural addressing scheme for each dyadic square $B \in \mathcal{G}_{k}$. The address has length $k$ and the symbols are from $\{0,1,2,3\}$; we write it as $\omega(B)=\left\{\omega_{i}(B)\right\}_{i=1}^{k}$. Recall that we arrange all dyadic squares in a tree. The construction of the random set is such that at even levels we take all children, but at odd levels we choose for each remaining square 
one child, uniformly at random and independently of the choices in other squares. This yields a subtree of the full 4-ary tree, where the nodes at level $2 n$ correspond to the random set $\mathcal{R}_{n}$.

By symmetry, we may assume that the slope of $\ell$ is positive.

Fix a small positive constant $\delta$, to be chosen later. We subdivide $\mathcal{G}_{2 n}$ into three types as follows:

(i) Say that $B \in \mathcal{G}_{2 n}$ is Type $\mathbf{1}$ if

$$
\#\left\{i \leq n-1: \omega_{2 i+1}(B)=0\right\} \geq \delta n .
$$

(ii) Say that $B \in \mathcal{G}_{2 n}$ is Type 2 if it is not Type 1 , and

$$
\#\left\{i \leq n-1: \omega_{2 i+1}(B)=2\right\} \geq \delta n .
$$

(iii) All remaining $B \in \mathcal{G}_{2 n}$ are said to be Type 3 .

Consider the events

$$
Z_{i}=\left\{\exists B \subset \mathcal{R}_{n}: B \in \text { Type } i \& B \cap \ell \neq \emptyset\right\} \text { for } i=1,2,3 .
$$

We have

$$
\mathbf{P}\left(\mathcal{R}_{n} \cap \ell \neq \emptyset\right) \leq \sum_{i=1}^{3} \mathbf{P}\left(Z_{i}\right) .
$$

First we estimate $\mathbf{P}\left(Z_{1}\right)$. We have

$$
\mathbf{E}\left[\#\left\{B \subset \mathcal{R}_{n}: B \cap \ell \neq \emptyset\right\} \mid Z_{1}\right] \leq \frac{\mathbf{E}\left[\#\left\{B \subset \mathcal{R}_{n}: B \cap \ell \neq \emptyset\right\}\right]}{\mathbf{P}\left(Z_{1}\right)} .
$$

Writing

$$
\#\left\{B \subset \mathcal{R}_{n}: B \cap \ell \neq \emptyset\right\}=\sum_{B \in \mathcal{G}_{2 n}} \mathbf{1}_{\left\{B \subset \mathcal{R}_{n}: B \cap \ell \neq \emptyset\right\}}
$$

and using that $\mathbf{P}\left(B \subset \mathcal{R}_{n}\right)=4^{-n}$ for any $B \in \mathcal{G}_{2 n}$, we obtain by (6.1) that

$$
\mathbf{E}\left[\#\left\{B \subset \mathcal{R}_{n}: B \cap \ell \neq \emptyset\right\}\right]=A_{n}(\ell) \cdot 4^{-n} \leq 2 .
$$

Thus, it remains to estimate the left-hand side of (6.4) from below. Let

$$
\Psi_{1}:=\left\{Q \in \mathcal{G}_{2 n}: Q \in \text { Type } 1 \& Q \cap \ell \neq \emptyset\right\} .
$$

Order the squares in $\mathcal{G}_{2 n}$ hit by $\ell$ from left to right and from bottom to top. This is a total order by the assumption on slope of the line $\ell$. For $Q \in \Psi_{1}$ consider the event

$$
Y_{Q}=\left\{Q \text { is the first square in } \Psi_{1} \text { hit by } \ell\right\} .
$$

Then $Z_{1}=\bigcup_{Q \in \Psi_{1}} Y_{Q}$ is a disjoint union, and so, for any random variable $f$,

$$
\mathbf{E}\left[f \mid Z_{1}\right]=\sum_{Q \in \Psi_{1}} \frac{\mathbf{P}\left(Y_{Q}\right)}{\mathbf{P}\left(Z_{1}\right)} \mathbf{E}\left[f \mid Y_{Q}\right] \geq \min _{Q \in \Psi_{1}} \mathbf{E}\left[f \mid Y_{Q}\right]
$$


Fix $Q \in \Psi_{1}$. We have

$$
\mathbf{E}\left[\#\left\{B \subset \mathcal{R}_{n}: B \cap \ell \neq \emptyset\right\} \mid Y_{Q}\right]=\sum_{B \in \mathcal{G}_{2 n}: B \cap \ell \neq \emptyset} \mathbf{P}\left(B \subset \mathcal{R}_{n} \mid Y_{Q}\right) .
$$

By the definition of Type 1 squares,

$$
\#\left\{i \leq n-1: \omega_{2 i+1}(Q)=0\right\} \geq \delta n .
$$

Fix $i$ such that $\omega_{2 i+1}(Q)=0$, and denote by $\widetilde{Q}$ the dyadic square in $\mathcal{G}_{2 i}$ having the address $\omega(\widetilde{Q})=\omega_{1}(Q) \ldots w_{2 i}(Q)$. The fact that $Q \subset \mathcal{R}_{n}$ implies that $\widetilde{Q}$ was chosen at the $i$ th stage of the random construction, i.e., $\widetilde{Q} \subset \mathcal{R}_{i}$. (Note that by definition, $[0,1]^{2} \supset \mathcal{R}_{1} \supset \ldots \supset \mathcal{R}_{n}$.) Since the slope of $\ell$ is positive, $\ell$ intersects at least $\frac{1}{2} 4^{n-i}$ squares $B \in \mathcal{G}_{2 n}$ whose addresses start with $\omega(\widetilde{Q}) k$, for $k \in\{1,2,3\}$ (see Figure 3). For each of these squares we have (using the independence of $Y_{Q}$ from the random choices involving the descendants of $\omega(\widetilde{Q}) k$ with $k \in\{1,2,3\})$, that

$$
\mathbf{P}\left(B \subset \mathcal{R}_{n} \mid Y_{Q}\right)=\mathbf{P}\left(B \subset \mathcal{R}_{n} \mid \widetilde{Q} \subset \mathcal{R}_{i}\right)=4^{i-n} .
$$

Therefore, the sum of $\mathbf{P}\left(B \subset \mathcal{R}_{n} \mid Y_{Q}\right)$ over the set of squares

$\mathcal{B}_{i}=\left\{B \in \mathcal{G}_{2 n},: B \cap \ell \neq \emptyset, \quad\left\{\omega_{j}(B)\right\}_{1}^{2 i}=\left\{\omega_{j}(Q)\right\}_{1}^{2 i}, \omega_{2 i+1}(B) \in\{1,2,3\}\right\}$, is at least $\frac{1}{2} 4^{n-i} \cdot 4^{i-n}=\frac{1}{2}$. Notice that the sets $\mathcal{B}_{i}$ are disjoint for distinct $i$ with $\omega_{2 i+1}(Q)=0$. Thus, the right-hand side of (6.6) is at least $\frac{1}{2} \delta n$, which, together with (6.7), (6.6), (6.5) and (6.4), implies

$$
\mathbf{P}\left(Z_{1}\right) \leq \frac{4}{\delta n} .
$$

By symmetry, we obtain

$$
\mathbf{P}\left(Z_{2}\right) \leq \frac{4}{\delta n}
$$

It remains to estimate $\mathbf{P}\left(Z_{3}\right)$. We have

$$
\begin{aligned}
\mathbf{P}\left(Z_{3}\right) & \leq \mathbf{E}\left[\#\left\{B \subset \mathcal{R}_{n}: B \in \text { Type } 3 \& B \cap \ell \neq \emptyset\right\}\right] \\
& =\sum_{B \in \text { Type } 3: B \cap \ell \neq \emptyset} \mathbf{P}\left(B \subset \mathcal{R}_{n}\right) \\
& =4^{-n} \#\{B \in \text { Type } 3: B \cap \ell \neq \emptyset\} .
\end{aligned}
$$

Thus, it suffices to bound the number of Type 3 squares hit by $\ell$. Consider the subtree of all dyadic squares that are hit by $\ell$. Since we assumed that $\ell$ does not hit any vertices, it can hit at most three children of a dyadic square that it intersects. For a Type 3 square, at least $n-2 \delta n$ of the digits at odd levels are either 1 or 3 , and our assumption that the slope of $\ell$ is positive 
guarantees that it cannot intersect both of the children labeled by 1 and 3 of any dyadic square (see Figure 3). Therefore, summing over the number

$$
j=\#\left\{i \leq n-1: \omega_{2 i+1}(B) \in\{0,2\}\right\},
$$

we obtain

$$
\#\{B \in \text { Type } 3: B \cap \ell \neq \emptyset\} \leq \sum_{j \leq 2 \delta n}\left(\begin{array}{c}
n \\
j
\end{array}\right) 3^{n} 2^{j} \leq C_{2} \cdot(1+\varepsilon(\delta))^{n} 3^{n+2 \delta n},
$$

where $\varepsilon(\delta) \rightarrow 0$, as $\delta \rightarrow 0$. Now we can choose $\delta$ so that $(1+\varepsilon(\delta)) \cdot 3^{1+2 \delta}<3.5$, and, in view of (6.10),

$$
\mathbf{P}\left(Z_{3}\right) \leq \text { const } \cdot(7 / 8)^{n} .
$$

Combining this with (6.8) and (6.9) yields (6.2), and the proof is complete.

\section{Concluding remarks and problems.}

7.1. More general families of self-similar sets. Theorems 2.1 and 2.3 extend to parametrized families of self-similar sets satisfying the "transversality condition." The following set-up is taken from [18].

Let $J \subset \mathbb{R}$ be a closed interval. Consider a one-parameter family of iterated function systems $\left\{S_{1}^{\lambda}, \ldots, S_{m}^{\lambda}\right\}_{\lambda \in J}$ where $S_{i}^{\lambda}(x)=r_{i} x+a_{i}(\lambda)$, with $r_{i} \in(0,1)$ and $a_{i}(\lambda) \in C^{1}(J)$. Let $\Pi(\lambda, \cdot): \mathcal{A}^{\mathbb{N}} \rightarrow \mathbb{R}$ be the natural projection map associated with the system and let $\Lambda^{\lambda}=\Pi\left(\lambda, \mathcal{A}^{\mathbb{N}}\right)$. Then $\left\{\Lambda^{\lambda}\right\}_{\lambda \in J}$ is a family of self-similar sets on the real line. Note that the similarity dimension $s$ does not depend on $\lambda$. We denote $f_{\omega, \tau}(\lambda)=\Pi(\lambda, \omega)-$ $\Pi(\lambda, \tau)$ and say that the transversality condition holds on $J$ if for any $\omega, \tau \in \mathcal{A}^{\mathbb{N}}$,

$$
\text { if } \exists \lambda \in J: f_{\omega, \tau}(\lambda)=f_{\omega, \tau}^{\prime}(\lambda)=0 \quad \text { then } \quad \omega=\tau .
$$

Define

$$
\mathcal{I} P=\left\{\lambda \in J: \exists \omega, \tau \in \mathcal{A}^{\mathbb{N}}: f_{\omega, \tau}(\lambda)=0 \text { but } \omega \neq \tau\right\} .
$$

Theorem 7.1. Suppose that the one-parameter family of iterated function systems defined above satisfies the transversality condition and $\mathcal{I} P=J$.

(i) Assume that $s=1$. Then there exist $C, a>0$ such that

$$
\int_{J}\left|\Lambda^{\lambda}(\rho)\right| d \lambda \leq C \exp \left[-a \log _{*}\left(\rho^{-1}\right)\right] \text { for all } \rho>0 .
$$

(ii) Assume that $s \leq 1$. Then $\mathcal{H}^{\phi}\left(\Lambda^{\lambda}\right)=0$ for Lebesgue-a.e. $\lambda \in J$ where $\phi(t)=t^{s} \exp \left[L \log _{*}\left(t^{-1}\right)\right]$, with $L \in(0, \log 2)$. 
The proof of this theorem is very similar to the proofs of Theorems 2.1 and 2.3. The only change is in Lemma 3.2, where one needs to use the general form of transversality rather than the special form (4.1) valid for projection families. In [18] it is proved, under the assumptions of Theorem 7.1(ii), that $\mathcal{H}^{s}\left(\Lambda^{\lambda}\right)=0$ for a.e. $\lambda \in J$.

Example. Let $\Lambda^{\lambda}=\left\{\sum_{n=0}^{\infty} a_{n} 4^{-n}: a_{n} \in\{0,1,2, \lambda\}\right\}$. Then all the assumptions of Theorem 7.1 hold for $\lambda \in[0,3]$.

7.2. Cantor sets with varying contraction ratios. Let $D=\left\{b_{1}, \ldots\right.$, $\left.b_{m}\right\} \subset \mathbb{R}^{2}$ be a digit set. Suppose that $\delta_{n} \geq 0$ and let

$$
r^{(n)}=m^{-n} \prod_{i=1}^{n}\left(1+\delta_{i}\right) \quad \text { for } n \geq 1 .
$$

Define $\Pi: \mathcal{A}^{\mathbb{N}} \rightarrow \mathbb{R}^{2}$ by $\Pi(\omega)=\sum_{n=1}^{\infty} r^{(n-1)} b_{\omega_{n}}$ and consider the set $\Lambda=\Pi\left(\mathcal{A}^{\mathbb{N}}\right)$. If $\delta_{n}=0$ for all $n$, then $\Lambda$ is self-similar, but we now assume that $\delta_{n}>0$ and $\delta_{n} \downarrow 0$. Further, suppose that

$$
\min _{i \neq j}\left|b_{i}-b_{j}\right| \cdot r^{(1)}>\max _{i, j}\left|b_{i}-b_{j}\right| \cdot \sum_{n=2}^{\infty} r^{(n)} .
$$

Then it is easy to see that $\Pi$ is one-to-one and so $\Lambda$ is a planar Cantor set. One can show that if the product $\prod_{i=1}^{\infty}\left(1+\delta_{i}\right)$ diverges, then the onedimensional Hausdorff measure of $\Lambda$ is not $\sigma$-finite (this follows, e.g., from applying the results of [20] to the natural measure on $\Lambda$ ). It turns out that if this product diverges sufficiently slowly, then $\operatorname{Fav}(\Lambda)=0$.

(Other deterministic sets of non- $\sigma$-finite $\mathcal{H}^{1}$ measure but zero Favard length can be found in $[\mathbf{1 3}, \mathbf{7}, \mathbf{8}]$.)

Proposition 7.2. There exists $c>0$ such that if

$$
\prod_{i=1}^{n}\left(1+\delta_{i}\right) \leq \exp \left[c \log _{*} n\right]
$$

then $\operatorname{Fav}(\Lambda)=0$.

Sketch of the proof. The argument closely follows the proof of Theorem 2.3 (in the homogeneous case), so we only give a brief sketch.

Let $\Pi_{\theta}=\operatorname{proj}_{\theta} \circ \Pi$ and $\Lambda_{u}^{\theta}=\Pi_{\theta}([u])$ where $[u]$ is the cylinder set corresponding to $u \in \mathcal{A}^{*}$. For $u, v \in \mathcal{A}^{*}$, with $|u|=|v|=n$, we say that $\Lambda_{u}^{\theta}$ and $\Lambda_{v}^{\theta}$ are $\varepsilon$-relatively close if the Hausdorff distance between these sets is not greater than $\varepsilon r^{(n)}$. Define $\Psi(n, k, \varepsilon)$ as the set of $\theta \in[0, \pi]$ such that there is no collection of distinct words $u_{1}, \ldots, u_{k}$, with $\left|u_{j}\right| \leq n$ for $j \leq k$, such that $\Lambda_{u_{j}}^{\theta}, j \leq k$, are pairwise $\varepsilon$-relatively close. The four lemmas in Section 3 and the proof of Theorem 2.3 (specialized to the homogeneous case $r_{i}=r$ ) go through essentially unchanged, replacing only $r^{n}, r^{q}$, etc., with $r^{(n)}, r^{(q)}$, etc. 
We use $\phi(t)=t$, so that $\mathcal{H}^{\phi}\left(\Lambda^{\theta}\right)=0$ for a.e. $\theta$ is equivalent to $\operatorname{Fav}(\Lambda)=0$. Further details are left to the reader.

\subsection{Unsolved problems.}

Question 7.3. For a one-dimensional self-similar set in the plane, which satisfies strong separation, can the bound (2.3) be strengthened to

$$
\operatorname{Fav}(\Lambda(\rho)) \leq C\left(\log \left(\frac{1}{\rho}\right)\right)^{-1} \text { for all } \rho>0,
$$

for some $C<\infty$ ?

Perhaps a more accessible goal is to improve our estimates for random Cantor sets.

Question 7.4. For the random sets $\mathcal{R}_{n}$ considered in Theorem 2.2, can the upper bound (2.5) be improved to

$$
\limsup _{n \rightarrow \infty} n \cdot \operatorname{Fav}\left(\mathcal{R}_{n}\right)<\infty \quad \text { a.s. ? }
$$

A more ambitious program would be to relate the decay rate of Favard length of neighborhoods, to other quantitative measures of nonrectifiability. The following question is motivated by Jones' Traveling Salesman Theorem $[6]$. Given a compact planar set $\Lambda$, and $\epsilon>0$, let

$$
\ell(\Lambda, \epsilon)=\sup \mathcal{H}_{\infty}^{1}(\Gamma(\epsilon) \cap \Lambda),
$$

where $\Gamma$ runs over recifiable curves of length 1 , and $\mathcal{H}_{\infty}^{1}$ denotes one-dimensional Hausdorff content. We can show that the four-corner set $K^{2}$ considered in the introduction satisfies $\ell\left(K^{2}, \epsilon\right)=O\left(|\log \epsilon|^{-1}\right)$ as $\epsilon \rightarrow 0$.

Question 7.5. Is there a quantitative estimate of $\operatorname{Fav}(\Lambda(\epsilon))$ in terms of $\ell(\Lambda, \epsilon)$ ?

In particular, is $\operatorname{Fav}(\Lambda(\epsilon))=O(\ell(\Lambda, \epsilon))$ as $\epsilon \rightarrow 0$ ?

Acknowledgments. The impetus for this work came from our joint paper with M. Rams and K. Simon [17] on the Hausdorff measure of self-conformal sets. We are grateful to them, and to P. Mattila, for useful discussions. Part of this work was done while the first author was visiting Microsoft Research. 


\section{References}

[1] C. Bandt and S. Graf, Self-similar sets 7. A characterization of self-similar fractals with positive Hausdorff measure, Proc. Amer. Math. Soc., 114 (1992), 995-1001, MR 93d:28014, Zbl 0823.28003.

[2] A.S. Besicovitch, Tangential properties of sets and arcs of infinite linear measure, Bull. Amer. Math. Soc., 66 (1960), 353-359, MR 22 \#11090, Zbl 0093.35801.

[3] G. David, Analytic capacity, Calderón-Zygmund operators, and rectifiability, Publ. Mat., 43 (1999), 3-25, MR 2000e:30044.

[4] K.J. Falconer, The geometry of fractal sets, Cambridge Tracts in Mathematics, 85, C.U.P., Cambridge-New York, 1986, MR 88d:28001, Zbl 0587.28004.

[5] J.E. Hutchinson, Fractals and self-similarity, Indiana Univ. Math. J., 30 (1981), 713747, MR 82h:49026, Zbl 0598.28011.

[6] P.W. Jones, Rectifiable sets and the travelling salesman problem, Invent. Math., 102 (1990), 1-15, MR 91i:26016, Zbl 0731.30018.

[7] P.W. Jones and T. Murai, Positive analytic capacity but zero Buffon needle probability, Pacific J. Math., 133 (1988), 99-114, MR 89m:30050, Zbl 0653.30016.

[8] H. Joyce and P. Mörters, A set with finite zero curvature and projections of zero length, J. Math. Anal. Appl., 247 (2000), 126-135, MR 2001j:28006, Zbl 0973.30022.

[9] R. Kenyon, Projecting the one-dimensional Sierpinski gasket, Israel J. Math., 97 (1997), 221-238, MR 98i:28002, Zbl 0871.28006.

[10] J.C. Lagarias and Y. Wang, Tiling the line with translates of one tile, Invent. Math., 124 (1996), 341-365, MR 96i:05040, Zbl 0847.05037.

[11] R. Lyons, Random walks, capacity and percolation on trees, Ann. Probab., 20 (1992), 2043-2088, MR 93k:6017, Zbl 0766.60091.

[12] J. Mateu, X. Tolsa and J. Verdera, On the semiadditivity of analytic capacity and planar Cantor sets, preprint, 2002.

[13] P. Mattila, Smooth maps, null-sets for integralgeometric measures and analytic capacity, Ann. Math., 123 (1986), 303-309, MR 87d:28010, Zbl 0589.28006.

[14] _ Orthogonal projections, Riesz capacities and Minkowski content, Indiana Univ. Math. J., 39 (1990), 185-198, MR 91d:28018, Zbl 0682.28003.

[15] _ Geometry of Sets and Measures in Euclidean Spaces, Cambridge University Press, 1995, MR 96h:28006, Zbl 0819.28004.

[16] On the analytic capacity and curvature of some Cantor sets with non- $\sigma$-finite length, Publ. Mat., 40(1) (1996), 195-204, MR 97d:30052, Zbl 0888.30026.

[17] Y. Peres, M. Rams, K. Simon and B. Solomyak, Equivalence of positive Hausdorff measure and the open set condition for self-conformal sets, Proc. Amer. Math. Soc., 129(9) (2001), 2689-2699 (electronic), MR 2002d:28004.

[18] Y. Peres, K. Simon and B. Solomyak, Self-similar sets of zero Hausdorff measure and positive packing measure, Israel J. Math., 117 (2000), 353-379, MR 2001g:28017, Zbl 0963.28008.

[19] Y. Peres and B. Solomyak, Approximation by polynomials with coefficients \pm 1 , J. Number Theory, 84(2) (2000), 185-198, CMP 1795789.

[20] C.A. Rogers and S.J. Taylor, Functions continuous and singular with respect to a Hausdorff measure, Mathematika, 8 (1962), 1-31, MR 24 \#A200, Zbl 0145.28701. 
Received October 31, 2000 and revised May 9, 2001. Research of Peres was partially supported by NSF grant \#DMS-9803597, and by the Landau center for Mathematical Analysis at the Hebrew University. Research of Solomyak was supported in part by NSF grant \#DMS 9800786 and the Institute of Mathematics at the Hebrew University.

Department of Mathematics

HEBREW UNIVERSITY

JERUSALEM

Department of Statistics

UNIVERSity OF CALIFORNIA

BERKELEY, CA 94720

E-mail address: peres@stat.berkeley.edu

Department of Mathematics, Box 354350

UNIVERSITY OF WASHINGTON

SEATTLE, WA 98195

E-mail address: solomyak@math.washington.edu 